\title{
Perfil epidemiológico da Sífilis gestacional e sua influência na transmissão vertical no Município de Parnamirim-RN
}

\author{
Epidemiological profile of gestational syphilis and its influence on vertical transmission in the City \\ of Parnamirim-RN \\ Perfil epidemiológico de la sífilis gestacional y su influencia en la transmisión vertical en el \\ Municipio de Parnamirim-RN
}

Recebido: 28/07/2021 | Revisado: 04/08/2021 | Aceito: 05/08/2021 | Publicado: 10/08/2021

\author{
Severino Azevedo de Oliveira Júnior \\ ORCID: https://orcid.org/0000-0001-8708-8794 \\ Universidade Federal do Rio Grande do Norte, Brasil \\ E-mail: juniorazevedo3719@yahoo.com.br \\ Elem Cristina Rodrigues Chaves \\ ORCID: https://orcid.org/0000-0002-6252-6361 \\ Centro Universitário Fibra, Brasil \\ E-mail: elemcrc@gmail.com \\ Matheus Barbosa Santos \\ ORCID: https://orcid.org/0000-0003-4622-6067 \\ Universidade Federal do Rio Grande do Norte, Brasil \\ E-mail: matheusifrn13@gmail.com \\ Ilka Lorena de Oliveira Farias Costa \\ ORCID: https://orcid.org/0000-0002-9816-5510 \\ Fundação Santa Casa de Misericórdia do Pará, Brasil \\ E-mail: ilka_lorena@yahoo.com.br \\ Daniele Socorro de Brito Souza Paiva \\ ORCID: https://orcid.org/0000-0003-4962-970X \\ Fundação Santa Casa de Misericórdia do Pará, Brasil \\ E-mail: dsbspaiva@gmail.com \\ Ana Paula Oliva Reis \\ ORCID: https://orcid.org/0000-0003-0354-6060 \\ Fundação Santa Casa de Misericórdia do Pará, Brasil \\ E-mail: anapaulaolivareis@gmail.com \\ Maria Helena Rodrigues de Mendonça \\ ORCID: https://orcid.org/0000-0003-0571-0565 \\ Centro Universitário Metropolitano da Amazônia, Brasil \\ E-mail: helenarmendonca@gmail.com \\ Sérgio Beltrão de Andrade Lima \\ ORCID: https://orcid.org/0000-0002-9531-2482 \\ Centro Universitário Metropolitano da Amazônia, Brasil \\ E-mail: sergio.lima@outlook.com
}

\begin{abstract}
Resumo
Objetivo: Analisar o perfil epidemiológico dos casos de sífilis gestacional no Município de Parnamirim - RN e os fatores predispostos à incidência da sífilis congênita, no período de 2010 a 2019. Metodologia: Estudo observacional e descritivo dos indicadores: taxa de detecção/1000 nascidos vivos da sífilis gestacional e congênita; perfil em gestantes e a situação assistencial no Pré-Natal. Resultados: Foi observada oscilação dos casos durante o período, sendo a sífilis gestacional, com máxima de 11,7/1000 em 2018 e congênita, com 2,3/1.000 em 2019. Houve maior frequência na faixa etária de 20 a 29 anos totalizando 51\%, de nível fundamental incompleto (39,4\%), idade gestacional de diagnóstico no terceiro trimestre $(44,1 \%)$ e classificação clínica para sífilis latente (53\%). Ao modelo assistencial, foi frequente à realização do Pré-Natal, com máxima de 88,5\%; ao período diagnóstico no parto e pósparto $(52,9 \%)$ e, quanto ao esquema de tratamento, $76 \%$ foram inadequados. Referente ao indicador de APS apresenta decréscimo durante o período, com 61,7\% para cobertura de ESF e 72,5\% para AB em 2019. Conclusão: Foi observado oscilação dos casos de sífilis, expondo lacunas no controle da doença, e falhas no modelo assistencial da Atenção Primária disposto no município, sobretudo, durante Pré-Natal, portanto, aumentando os riscos de transmissão vertical para sífilis.
\end{abstract}

Palavras-chave: Sífilis; Sífilis congênita; Assistência materno-infantil; Vigilância epidemiológica.

\footnotetext{
Abstract

Objective: To analyze the epidemiological profile of cases of gestational syphilis in the city of Parnamirim - NB and the factors predisposed to the incidence of congenital syphilis, from 2010 to 2019. Methodology: Observational and
} 
descriptive study of the indicators: detection rate/1000 live births of gestational and congenital syphilis; profile in pregnant women and the care situation in prenatal care. Results: It was observed oscillation of the cases during the period, with gestational syphilis, with maximum of 11.7/1000 in 2018 and congenital, with 2.3/1,000 in 2019. There was a higher frequency in the 20 to 29 years age group, totaling 51\%, incomplete elementary level $(39.4 \%)$, gestational age of diagnosis in the third trimester (44.1\%) and clinical classification for latent syphilis (53\%). In the care model, prenatal care was frequently performed, with a maximum of $88.5 \%$; in the period of diagnosis during delivery and postpartum (52.9\%); and, regarding the treatment schedule, $76 \%$ were inadequate. Regarding the PHC indicator, there was a decrease during the period, with $61.7 \%$ for FHS coverage and $72.5 \%$ for PC in 2019. Conclusion: We observed oscillation of syphilis cases, exposing gaps in the control of the disease, and failures in the primary care model arranged in the municipality, especially during prenatal care, thus increasing the risks of vertical transmission for syphilis.

Keywords: Syphilis; Congenital syphilis; Maternal and child care; Epidemiological surveillance.

\section{Resumen}

Objetivo: Analizar el perfil epidemiológico de los casos de sífilis gestacional en el Municipio de Parnamirim - RN y los factores predispuestos a la incidencia de la sífilis congénita, en el período de 2010 a 2019. Metodología: Estudio observacional y descriptivo de los indicadores: tasa de detección/1000 nacidos vivos de la sífilis gestacional y congénita; perfil en gestantes y la situación asistencial en el Prenatal. Resultados: Se observó oscilación de los casos durante el período, siendo la sífilis gestacional, con máxima de 11,7/1000 en 2018 y congénita, con 2,3/1.000 en 2019. Hubo mayor frecuencia en la franja etaria de 20 a 29 años totalizando 51\%, de nivel fundamental incompleto (39,4\%), edad gestacional de diagnóstico en el tercer trimestre $(44,1 \%)$ y clasificación clínica para sífilis latente $(53 \%)$. Al modelo asistencial, fue frecuente la realización del Prenatal, con máxima de 88,5\%; al período diagnóstico en el parto y postparto $(52,9 \%)$ y, en cuanto al esquema de tratamiento, $76 \%$ fueron inadecuados. Referente al indicador de APS presenta disminución durante el período, con 61,7\% para cobertura de ESF y 72,5\% para AB en 2019. Conclusión: Se observó oscilación de los casos de sífilis, exponiendo brechas en el control de la enfermedad, y fallas en el modelo asistencial de la Atención Primaria dispuesto en el municipio, sobre todo, durante Prenatal, por lo tanto, aumentando los riesgos de transmisión vertical para sífilis.

Palabras-clave: Sífilis; Sífilis congénita; Asistencia materno-infantil; Vigilancia epidemiológica.

\section{Introdução}

A sífilis é uma patologia infectocontagiosa, crônica, com manifestações cutâneas e sistêmicas considerava um grave problema de saúde pública mundial, principalmente nos países em desenvolvimento, sobretudo, pelas consequências sanitária, econômicas e sociais em decorrência aos desafios de prevenção, acesso aos serviços de saúde, diagnóstico precoce e tratamento oportuno, os quais inferem diretamente em um prognóstico inadequado (Brasil, 2020; Gonçalves et al., 2021; Miranda et al., 2021).

Causada pela bactéria Treponema pallidum, a sífilis é uma doença de notificação compulsória desde 1986 transmitida, predominantemente, por via sexual (oral, vaginal e anal), portanto, uma Infecção Sexualmente Transmissível (IST). Contudo, quando estabelecida durante a gestação, há possibilidade de transmissão vertical, caracterizando a sífilis congênita, a qual pode levar ao aborto espontâneo, prematuridade, comprometimento oftalmológico, auditivo e neurológico e manifestações clínicas precoces ou tardias da sífilis congênita, além de expor um coeficiente de mortalidade fetal acima de 40\%, portanto, a partir de sua elevada morbimortalidade infantil, a sífilis gestacional é considerada de notificação compulsória desde 2005 e o diagnostico e tratamento precoce são medidas efetivas para evitar a transmissão vertical (Brasil, 2020; Freitas et al., 2021).

Segundo Nunes e Barbosa (2020), a taxa de transmissão vertical varia de $80 \%$ a $100 \%$ seguindo o estágio da sífilis materna durante a gestação e a duração da exposição fetal: sífilis primária (70-100\%), sífilis secundária (90\%), latente precoce (40\%), e latente tardia (10\%), ou seja, quanto mais avançada a doença materna, menor é o risco de transmissão e a cada gestação sucessiva, a chance de transmissão diminui, contudo, sem eliminar os riscos.

A Assembleia Mundial de Saúde, em 2016, estabeleceu a estratégia global de saúde às Infecções Sexualmente Transmissíveis (IST) entre 2016 a 2021, visando à expansão das intervenções, ações e serviços para controlar as IST's e diminuir seus impactos como um problema de saúde pública até 2030, contudo, de acordo com os dados da Organização Mundial da Saúde - OMS, em 2018 houve registro de mais de 12 milhões de casos e, em nível de Brasil, entre 2009 a 2018, 
foram diagnosticadas cerca de 247,583 casos de sífilis em gestantes, sendo 59,6\% com transmissão vertical e, em 2019, expôs 61.127 casos de sífilis em gestantes, com taxa de detecção de 20,8/1000 nascidos vivos, com incidência da sífilis gestacional de 8,2/1000 nascidos vivos e mortalidade de 5,9/1000 nascidos vivos (OMS, 2017; Brasil, 2020). Com isso, representando um dos maiores desafios dos sistemas de saúde mundial diante ao controle da sífilis e, tão logo, o agravamento do sistema público brasileiro e as necessidades dos esforços conjunto em nível de prevenção e tratamento da doença.

Nesse contexto, as ações de prevenção para transmissão vertical encontram-se estritamente coligadas aos cuidados existentes durante o acompanhamento e triagem no Pré-Natal e o tratamento oportuno e adequado tanto da gestante diagnosticada quanto do parceiro em um prazo máximo de 30 dias antes do parto, entretanto, é de conhecimento as lacunas existentes no sistema público de saúde como reflexo aos cortes orçamentários, os quais interferem no fortalecimento do SUS como estratégia de contenção às doenças (Lopes, 2020).

Destarte, considerando os desafios predispostos ao sistema de saúde e a alta incidência de sífilis em gestante e congênita, esse cenário é igualmente preocupante no município de Parnamirim, localizado no Estado do Rio Grande do Norte (RN) e, a partir da carência literária especificamente ao município, o desconhecimento do perfil epidemiológico da sífilis favorece as ações desfocadas e a incipiente prática considerando o processo de territorialização pelas equipes de saúde e, como consequência, tornando a rede de saúde meramente executora de ações de saúde não direcionadas as necessidades locais (Camargos e Oliver, 2019). A partir disso, sendo a Vigilância em Saúde (VS) instrumento e ferramenta de informação base para o planejamento em serviços de saúde, sobretudo, na Atenção Primária em Saúde (APS), o presente estudo tem como intuito contribuir à reorganização das ações de controle da sífilis gestacional e congênita no município de Parnamirim-RN, a partir das ações integradas na atenção básica e fornecer subsídio aos gestores para intervir na conjunção de fatores que fundamentam a incidência e prevalência da doença.

Portanto, tem por objetivo o analisar o perfil epidemiológico dos casos de sífilis gestacional no Município de Parnamirim - RN e os fatores predispostos à incidência da sífilis congênita, no período de 2010 a 2019.

\section{Metodologia}

Trata-se de um estudo observacional, com aspecto descritivo realizado no município de Parnamirim, Rio Grande do Sul, pertence à Região Metropolitana de Natal, à mesorregião do Leste Potiguar e à microrregião de Natal. Localizado no sul da capital estadual, com uma distância de apenas 12 quilômetros, o município ocupa uma área de $123 \mathrm{~km}^{2}$, e tem população estimada de 255.793 habitantes, portanto, o terceiro município mais populoso do Estado (IBGE, 2018). Detém em sua atenção primária comporta por 30 Unidades básicas de Saúde, 54 Estratégias de Saúde da Família (ESF), sendo 2 Estratégias de Saúde Prisional (ESP), 05 equipes de Núcleo de Apoio a Saúde da Família (NASF) e 01 equipe Consultório na Rua tipo II.

Os dados do estudo abrange uma análise quantitativa da série temporal dos indicadores: taxa de detecção/1.000 nascidos vivos de sífilis gestacional e congênita, dados básicos do perfil da sífilis em gestantes, considerando as variáveis: faixa etária, escolaridade, idade gestacional e classificação clínica, realização do pré-natal, período de diagnóstico da sífilis e esquema de tratamento materno, além de dados referentes aos Indicadores da Atenção Primária em Saúde: Cobertura Estratégia Saúde da Família e Cobertura Atenção Básica, no período de 2010 a 2019.

Os dados foram extraídos pelo Departamento de Doenças de Condições Crônicas e Infecções Sexualmente Transmissíveis do Ministério da Saúde (DASIS/MS), no período de 2010 a 2019 (atualizados em 06/2020). Para obtenção da taxa de detecção, os casos de sífilis gestacional e congênita foram padronizados segundo estimativa de população do Instituto Brasileiro de Geografia e Estatística - IBGE, tendo como numerador: a quantidade de casos no município por ano e, denominador: quantitativo de nascidos vivos x 1.000. Referente aos dados básicos da sífilis, foram representados sob forma de frequência relativa $(\%)$. 


\section{Taxa de Detecção =}

Quantitativo de casos de sífilis por ano

x 1.000

Quantitativo de nascidos vivos

Para obtenção dos Indicadores da Atenção Primária em Saúde, foi utilizada a plataforma de gestão: Informação e Gestão da Atenção Básica (e-Gestor AB), com dados expondo a média percentual das coberturas de ESF e AB no município, de 2010 a 2019. Para coleta e elaboração de gráficos e tabelas, foi utilizado o software Excel 2016.

\section{Resultados e Discussão}

De acordo com os dados obtidos pelo DASIS/MS em Parnamirim - RN, considerando a taxa de detecção por 1.000 nascidos vivos, no período de 2010 a 2019, foram registrados 267 casos de sífilis em gestante, expondo oscilação na taxa de detecção durante todo período, com mínima de 0,5/1000 em 2011 e 2014 e máxima de 11,7/1000 em 2018. E, referente à sífilis congênita, foram confirmados 208 casos em menores de 1 ano, com taxa de detecção oscilante, sendo de 2,3/1.000 em 2019 (Figura 1).

Figura 1 - Distribuição da taxa de detecção de sífilis em gestante e congênita por 1.000 nascidos vivos no município de Parnamirim - RN, de 2010 a 2019.

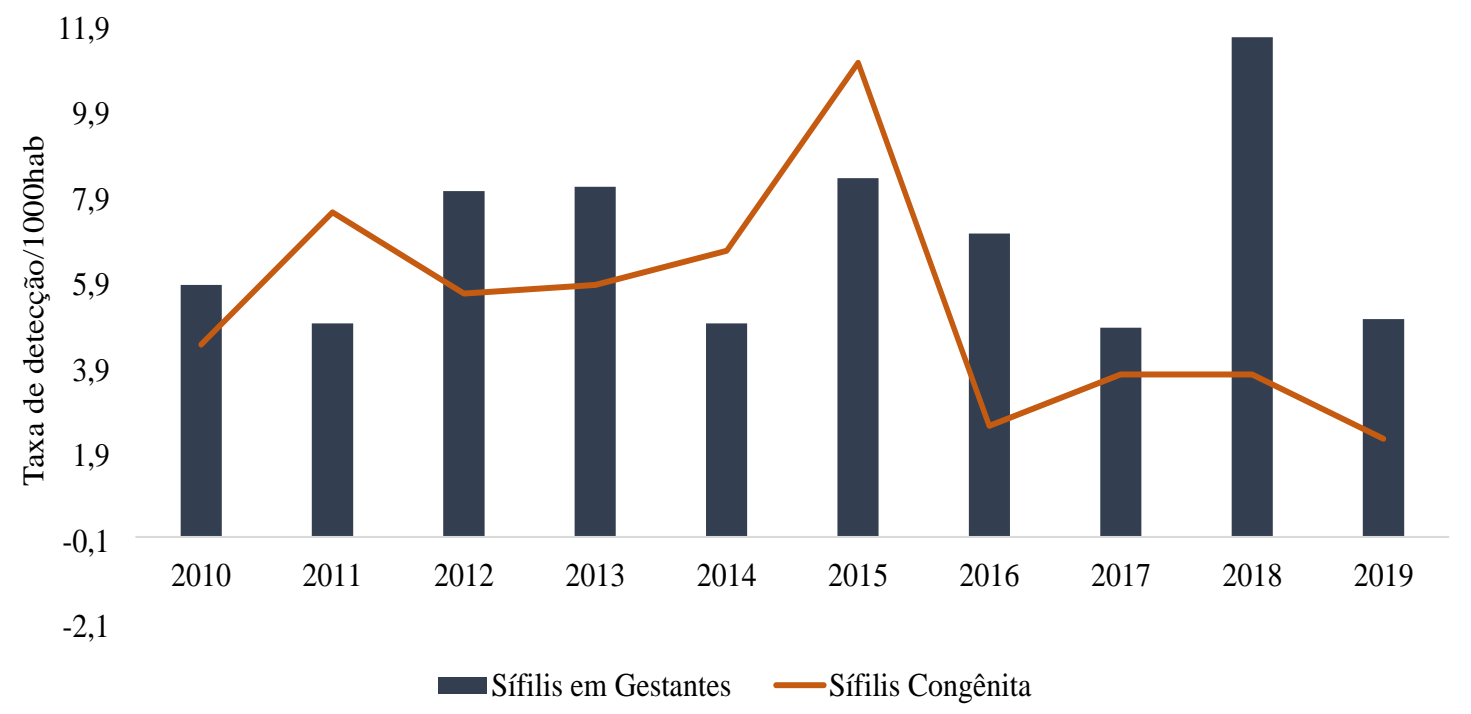

Fonte: MS/SVS/Departamento de Doenças de Condições Crônicas e Infecções Sexualmente Transmissíveis.

Segundo Junior et al (2019), a rede de saúde de Parnamirim/RN atua de acordo os Procedimentos Operacionais Padrões (POP) preconizados pelo MS onde, a partir do momento do diagnóstico de gestação, toda mulher deve realizar testagem rápida na primeira consulta de Pré-Natal e novamente no início do $3^{\circ}$ trimestre. Além disso, é de responsabilidade das Unidades de Saúde o monitoramento sorológico e, caso reagente o resultado para sífilis, garantir o tratamento adequado e em tempo oportuno com aplicação da penicilina benzatina, além o incentivar o parceiro da gestante às consultas posteriores, assim como o seu tratamento numa possível eventualidade. Todavia, apesar da visível diminuição dos casos em 2019, de acordo com os dados, é possível observar certo desafio e fragilidade ao controle da sífilis gestacional, tendo como reflexo a oscilação de casos no período estudado, e as dificuldades nas medidas de prevenção à transmissão vertical, uma vez que de 267 casos em gestantes, em $78 \%$ houve transmissão ao recém-nascido, logo, dados preocupantes devido ao alto grau de impacto à saúde da 
criança e da gestante.

Contudo, seguindo essa oscilação durante o período e a diminuição de casos em 2019, apesar das falhas e fragilidades do sistema público de saúde anteriormente descrito, Lima et al (2019) e Costa et al (2021) expõem que os indicadores epidemiológicos de um determinado município são base para planejar executar as ações de vigilância e os serviços dispostos na atenção básica sendo, portanto, essencial sua análise ampla considerando distintos contextos. Nesse caso, sendo também grandemente relatada na literatura, a subnotificação é um problema recorrente em diversos países, sobretudo, onde a integração entre VS e APS é incipiente, como no Brasil, logo, constitui-se como fator contributivo à persistência da sífilis como problema de saúde pública devendo, assim, ser considerando como influente ao perfil exposto (Tiago et al., 2017; Costa et al., 2020).

Destarte, dados da literatura expõe o mesmo perfil de casos em diferentes cidades do Rio Grande do Norte, em Natal/RN, a taxa de detecção da sífilis congênita foi crescente desde 2011, saindo de 6/1.000 para 14,8/1.000 nascidos vivos em 2015; seguindo essa mesma lógica em Caicó/RN, onde a taxa de incidência da sífilis congênita foi de 7,8/100mil em 2016 para 12,5/100mil nascidos vivos em 2018, com mortalidade de 10,8/100mil (Ferreira et al., 2018; Ferreira et al., 2021), caracterizando a epidemia da sífilis. Contudo, não são restritos ao estado, estudos realizados em São Paulo, Goiás e Paraná demonstram essa incidência crescente e, como justificativas, remetem o aumento da cobertura de testagem com a ampliação do uso de testes rápidos para distintas localidades, entretanto, expressam o tratamento tardio e/ou inadequado das gestantes (Donalísio et al., 2017; Neto et al., 2019; Pilger et al., 2019).

Destarte, de forma geral, segundo MS (2020), o RN apresenta-se com uma das maiores frequências de gestantes que não realizaram o tratamento para sífilis $(8,7 \%)$ e, como reflexo, está entre os estados com taxa de detecção de sífilis congênita maior que a média nacional, com 12,3/100mil e mortalidade de 3,8/100mil nascidos vivos. De acordo com Figueiredo et al (2020), embora entre 2011 e 2014 o Brasil tenha investido no aumento de 3.124.910 teste para triagem durante a gestação, a distribuição foi realizadas de forma heterogênea entre os estados, sobretudo, pertencentes a região Nordeste, revelando a desigualdade social e as dificuldades na superação das limitações do acesso ao diagnóstico e, além disso, o autor revela que menos de $50 \%$ dos municípios da região realizam a aplicação de penicilina no tratamento das gestantes reduzindo, portanto, o acesso ao tratamento de qualidade na atenção básica e, consequentemente, favorecendo a cadeia de transmissão, podendo inferir a isso o perfil encontrado nesse estudo e aos demais dispostos na região, logo, indo em contramão as recomendações das entidades nacionais e internacionais de saúde.

O MS (2018) instituiu em 2018 o Projeto de Resposta Rápida à Sífilis nas Redes de Atenção com objetivo de reduzir à sífilis adquirida, gestacional e congênita; fortalecer a vigilância epidemiológica; integrar a VS e APS nas redes de atenção e articular setores sociais e comunidades visando uma resposta rápida a sífilis, logo, implementada em abril de 2018 no município de Parnamirim/RN, o Conselho de Secretarias Municipais de Saúde do Rio Grande do Norte (Cosems-RN, 2018) relata avanço no diagnóstico e tratamento da Sífilis, todavia, os dados expõe um certo distanciamento do município diante as metas estabelecidas, além de estudos demonstrarem que os objetivos traçados são os principais desafios do Brasil, sobretudo, em nível de controle dos casos, integração dos serviços e qualidade da assistência na atenção básica, tendo como fator barreiras em diferentes níveis de atuação no setor da saúde (Macedo et al., 2020; Costa et al., 2020; 2021). Com isso, fundamenta a necessidade de estratégias diferenciadas visando o aprimoramento dos serviços em diferentes níveis de atenção a saúde, visando à caracterização da busca ativa e integralidade dos serviços, além do monitoramento constante das ações em saúde visando, de fato, contemplar os objetivos estabelecidos no projeto e sua conjuntura condizente com o escopo.

Analisando o perfil social associado ao diagnóstico de sífilis na gestação, no período de 2010 a 2019, foi observada maior frequência disposta na faixa etária de 20 a 29 anos em todo período, totalizando $51 \%$ dos casos; quanto à escolaridade, o mesmo perfil se mantém durante 2010-2019, com predomínio do nível fundamental incompleto, somando 39,4\% dos casos; referente à idade gestacional, obteve maior frequência o terceiro trimestre, somando $44,1 \%$ dos casos. Contudo, referente à 
classificação clínica, foi observado mudança de perfil a partir de 2014, saindo da maior frequência de sífilis primária para sífilis latente, representando um total de 53\% dos casos (Tabela 1).

Tabela 1 - Distribuição da frequência relativa (\%) da sífilis em gestante, segundo as variáveis sociais, no município de Parnamirim - RN, de 2010 a 2019.

\begin{tabular}{|c|c|c|c|c|c|c|c|c|c|c|}
\hline \multirow{2}{*}{$\frac{\text { Variáveis }}{\text { Faixa Etária }}$} & \multicolumn{10}{|c|}{ Parnamirim-RN } \\
\hline & 2010 & 2011 & 2012 & 2013 & 2014 & 2015 & 2016 & 2017 & 2018 & 2019 \\
\hline 10 a 14 & 0 & 0 & 3,3 & 3,1 & 0 & 0 & 7,4 & 0 & 0 & 0 \\
\hline 15 a 19 & 33,3 & 11,8 & 30 & 25 & 25 & 34,3 & 18,5 & 68,4 & 21,7 & 10 \\
\hline 20 a 29 & 42,9 & 52,9 & 46,7 & 62,5 & 55 & 40 & 51,9 & 31,6 & 58,7 & 45 \\
\hline 30 a 39 & 23,8 & 35,3 & 20 & 9,4 & 20 & 22,9 & 22,2 & 0 & 19,6 & 45 \\
\hline$>40$ & 0 & 0 & 0 & 0 & 0 & 2,9 & 0 & 0 & 0 & 0 \\
\hline \multicolumn{11}{|l|}{ Escolaridade } \\
\hline Fundamental Incompleto & 60 & 60 & 45 & 33,3 & 17,6 & 34,8 & 43,5 & 33,3 & 39,4 & 42,9 \\
\hline Fundamental Completo & 20,0 & 13,3 & 20,0 & 6,7 & 23,5 & 21,7 & 26,1 & 16,7 & 12,1 & 14,3 \\
\hline Médio Incompleto & 13,3 & 13,3 & 25 & 30 & 41,2 & 34,8 & 13 & 25 & 18,2 & 21,4 \\
\hline Médio Completo & 6,7 & 13,3 & 10 & 30 & 17,6 & 8,7 & 8,7 & 25 & 30,3 & 14,3 \\
\hline Superior Incompleto & 0 & 0 & 0 & 0 & 0 & 0 & 4,3 & 0 & 0 & 0 \\
\hline Superior Completo & 0 & 0 & 0 & 0 & 0 & 0 & 4,3 & 0 & 0 & 7,1 \\
\hline \multicolumn{11}{|l|}{ Idade Gestacional (trimestre) } \\
\hline $1^{\circ}$ Trimestre & 31,3 & 29,4 & 13,8 & 16,7 & 15,8 & 23,5 & 37,0 & 42,1 & 28,9 & 31,6 \\
\hline $2^{\circ}$ Trimestre & 37,5 & 35,3 & 6,9 & 16,7 & 52,6 & 47,1 & 33,3 & 26,3 & 20 & 21,1 \\
\hline $3^{\circ}$ Trimestre & 31,3 & 35,3 & 79,3 & 66,7 & 31,6 & 29,4 & 29,6 & 31,6 & 51,1 & 47,4 \\
\hline \multicolumn{11}{|l|}{ Classificação Clínica } \\
\hline Sífilis Primária & 76,9 & 64,3 & 43,5 & 78,9 & 5,3 & 14,7 & 30 & 23,5 & 22,5 & 5,9 \\
\hline Sífilis Secundária & 7,7 & 21,4 & 34,8 & 5,3 & 5,3 & 0 & 5 & 17,6 & 0 & 0 \\
\hline Sífilis Terciária & 15,4 & 14,3 & 21,7 & 5,3 & 0 & 0 & 5 & 0 & 5 & 11,8 \\
\hline Sífilis Latente & 0 & 0 & 0 & 10,5 & 89,5 & 85,3 & 60 & 58,8 & 72,5 & 82,4 \\
\hline
\end{tabular}

Fonte: MS/SVS/Departamento de Doenças de Condições Crônicas e Infecções Sexualmente Transmissíveis.

De acordo com Gonçalves et al (2021), não há um consenso alusivo a tais características diante aos fatores de risco para sífilis, todavia, o perfil social encontrado no presente estudo é constantemente relatado na literatura e reflete, especialmente, em uma maior chance de ocorrência nos desfechos analisados. Em seu estudo, Domingues e Leal (2016), expõem que $80 \%$ das gestantes com desfecho para sífilis congênita estiveram dispostas na faixa de 20 a 34 anos; para Nunes et al (2020) e Ferreira et al (2021), os dados são similares, com diagnóstico de sífilis durante a gestação predominantemente na faixa de 20 a 29 anos, com 52,2\% e 49,7\%, respectivamente. Indo em consenso com os dados, os mesmos autores inferem maior detecção em mães jovens com baixo nível de instrução: para Domingues e Leal (2016), 60,6\% estão presentes em fundamental incompleto para mães com desfecho de sífilis congênita e 38,8\% sem desfecho para sífilis congênita; Ferreira et al (2021), 37,4\% predominam em ensino de $5^{\circ}$ a $8^{\circ}$ séria incompleta.

A partir dos dados, se estabelece a maior vulnerabilidade social no público jovem de baixa escolaridade que, segundo Lima et al (2019) e Oliveira et al (2021), se justifica pela fase da vida em que a pratica sexual e o rotina cotidiana são intensas, logo, implicando em uma diminuição da procura pelos serviços em saúde, consequentemente, a não realização do Pré-Natal devido ao baixo nível de instrução acerca de sua importância e os riscos à transmissão vertical, além de sua contribuição à exposição das IST’s devido a limitada percepção sobre as medidas de prevenção. Portanto, apesar do perfil social apresentar variações na literatura, Nunes et al (2020) afirma que o baixo nível de instrução e a população jovem são marcadores de maior risco para exposição da sífilis gestacional e congênita. 
Ademais, apesar de não ser uma variável analisada no presente estudo, ressalta sua importância como fator à incidência de sífilis, logo, recorrente na literatura e diz respeito à questão renda onde, segundo Macedo et al (2017) e Motta et al (2018), população com menor condição econômica tende a expor constâncias nos números de casos, sendo justificadas pelos desafios e restrições de acesso e transporte às Unidades Básicas de Saúde (UBS) em busca de serviços. Segundo dados do IBGE (2018), o Rio Grande do Norte expõe o patamar recorde de desigualdade de renda, onde metade da população tem renda média mensal - per capita - de apenas $\mathrm{R} \$ 289$, expondo um índice Geni de 0,5 corroborando, portanto, os dados de maior vulnerabilidade e desigualdade social em saúde como suporte ao desfecho preocupante da sífilis no município de Parnamirim.

A partir disso, constitui-se como fundamentais e primordiais ações diferenciadas com objetivo de alcançar amplamente a populações, sobretudo, as mais vulneráveis com vista na universidade e equidade em saúde, e então promover a comunicação e o incentivo à informação e orientação em massa acerca da educação em saúde voltada à prática sexual protegida, planejamento familiar e os serviços de saúde a serem realizados durante período gestacional e, tão logo, proporcionar uma mudança de perfil (Domingues e leal, 2016; Ferreira et al., 2021; Oliveira et al., 2021).

Referente idade gestacional, a maior frequência apresenta-se no terceiro trimestre, sendo condizente com a literatura onde, segundo Conceição et al (2019), 46,3\% foram diagnosticadas no $3^{\circ}$ trimestre e, em estudo de Gonçalves et al (2020), a taxa de detecção da sífilis foi de 90,5/100 habitantes no $3^{\circ}$ trimestre, seguido do $2^{\circ}$ trimestre. De acordo com MS (2019), recomenda-se que seja realizado o mínimo de 6 consultas durante Pré-Natal, com início até a $13^{\circ}$ semana de gestação e, tão logo, a realização de teste sorológico para sífilis para então seguir ao tratamento da gestante e prevenção da transmissão.

No entanto, a partir dados, se fundamenta as discussões de Sanson et al (2018) e Nunes et al (2020) em que se constitui a problemática da baixa adesão à assistência ao Pré-Natal e a baixa sensibilidade e qualidade da assistência à gestante como justificativa à detecção tardia da sífilis ( $3^{\circ}$ trimestre) e, consequentemente, o aumento da possibilidade de transmissão, podendo inferir a isso a transmissão em $78 \%$ dos casos no município de Parnamirim/RN. Assim, embora as medidas de prevenção e tratamento seja relativamente simples e eficaz diante a redução da morbimortalidade associados à transmissão vertical, o perfil até então traçado evidencia entraves e deficiência dos serviços de saúde nas adequações às medidas de atenção, a detecção, diagnóstico precoce e o tratamento oportuno da gestante afim de um prognóstico satisfatório.

Diferentemente dos perfis relatados na literatura com maior frequência para sífilis primária, a transição da sífilis primária para latente é presente no município a partir de 2014, contudo, segundo MS (2019) essa frequência é esperada uma vez que a maioria dos diagnósticos ocorre nesse estágio e, quando a cronologia do tempo de infecção não é bem determinada (duração ignorada), se classifica e trata como sífilis latente (especificamente tardia), período onde não há manifestações clínicas da sífilis, porém, reatividade nos testes imunológicos. No entanto, de acordo com Nunes et al (2020), esse o aumento de casos com duração ignorada é reflexo do desconhecimento e dúvidas dos profissionais da saúde quanto o histórico da doença dificultando, portanto, o diagnóstico clínico da gestante. Logo, remete as capacitações de nível profissional com no diagnóstico e notificação adequados como uma estratégia representativa frente às lacunas existentes na deteç̧ão e fortalecimento das redes de atenção na caracterização dos casos.

Reconhecendo que o acompanhamento Pré-Natal, quando realizado de forma adequada, constitui uma importante ferramenta para redução da incidência da sífilis congênita, foi realizada uma análise indireta da situação assistencial no PréNatal. A partir dos dados do DASIS/MS em Parnamirim - RN quanto à realização do Pré-Natal, a maior frequência concentrase em sua realização durante o período, com mínima de 70\% e máxima de 88,5\%; ao período diagnóstico, expos o total de 47,1\% durante o Pré-Natal e 52,9\% no parto/curetagem e após o parto; quanto ao esquema de tratamento, total de $76 \%$ é inadequado. Referente ao indicador de APS, obtido pelo e-Gestor AB, as variáveis apresentam decréscimo durante o período, chegando em $61,7 \%$ para cobertura de ESF e 72,5\% para AB (Tabela2). 
Tabela 2 - Distribuição da frequência relativa (\%) da situação assistencial no Pré-Natal e Indicadores da Atenção Primária em Saúde no município de Parnamirim - RN, de 2010 a 2019.

\begin{tabular}{|c|c|c|c|c|c|c|c|c|c|c|}
\hline \multirow{2}{*}{$\begin{array}{c}\text { Variáveis } \\
\text { Realização do Pré-Natal }\end{array}$} & \multicolumn{10}{|c|}{ Parnamirim-RN } \\
\hline & 2010 & 2011 & 2012 & 2013 & 2014 & 2015 & 2016 & 2017 & 2018 & 2019 \\
\hline Sim & 87,5 & 88,5 & 81,0 & 87,0 & 88,0 & 86,7 & 70,0 & 78,6 & 85,7 & 77,8 \\
\hline Não & 12,5 & 11,5 & 19,0 & 13,0 & 12,0 & 13,3 & 30,0 & 21,4 & 14,3 & 22,2 \\
\hline \multicolumn{11}{|l|}{ Período Diagnóstico } \\
\hline Durante o pré-natal & 56,3 & 38,5 & 33,3 & 47,8 & 48,1 & 47,8 & 60 & 26,7 & 73,3 & 55,6 \\
\hline Parto/curetagem & 37,5 & 46,2 & 28,6 & 47,8 & 48,1 & 52,2 & 40 & 73,3 & 20 & 44,4 \\
\hline Após o parto & 6,3 & 15,4 & 38,1 & 4,3 & 3,7 & 0 & 0 & 0 & 6,7 & 0 \\
\hline \multicolumn{11}{|l|}{$\begin{array}{c}\text { Esquema de tratamento } \\
\text { materno }\end{array}$} \\
\hline Adequado & 0 & 0 & 0 & 0 & 0 & 0 & 0 & 0 & 0 & 11,1 \\
\hline Inadequado & 62,5 & 69,2 & 81 & 82,6 & 81,5 & 78,3 & 80 & 73,3 & 66,7 & 77,8 \\
\hline Não Realizado & 25 & 26,9 & 19 & 17,4 & 14,8 & 21,7 & 20 & 26,7 & 20 & 11,1 \\
\hline Ignorado & 12,5 & 3,8 & 0 & 0 & 3,7 & 0 & 0 & 0 & 13,3 & 0 \\
\hline \multicolumn{11}{|l|}{ Indicadores da APS } \\
\hline Cobertura ESF & 77,8 & 68,7 & 69,3 & 67,5 & 64,6 & 68,2 & 73,5 & 64,1 & 63,6 & 61,7 \\
\hline Cobertura AB & 100 & 97 & 94,9 & 81,6 & 71,4 & 77 & 82,3 & 74,9 & 79,3 & 72,5 \\
\hline
\end{tabular}

Fonte: MS/SVS/Departamento de Doenças de Condições Crônicas e Infecções Sexualmente Transmissíveis.

A partir dos dados, embora registo de maior frequência em gestantes que realizaram o Pré-Natal, corrobora a prevalência percentual e o aumento de 2018 (14,3\%) para 2019 (22,2\%) às que não realizaram, logo, dados preocupantes uma vez que essa assistência fundamenta a diminuição na incidência da sífilis congênita. Ademais, a análise dos dados expõe o cenário alarmante e debilitado do modelo assistencial na atenção básica disposto no município de Parnamirim/RN, onde apenas 47,1\% da sífilis são diagnosticadas durante o Pré-Natal e mais de 50\% no momento do parto e pós-parto, além da quase nula frequência de tratamento adequado, com apenas 11\% em 2019 e a diminuição dos indicadores de qualidade da atenção básica, portanto, o Pré-Natal permanece aquém da desejada, sendo foco para manutenção da infeção em recém-nascido.

Não é inédita a melhoria dos serviços de saúde, sobretudo, no âmbito assistencial materno-infantil, sendo esse um dos objetivos definidos pelo MS e fundamental para qualificar os serviços oferecidos no período gestacional até a puérpera assim, baseado nessa premissa, a Rede Cegonha emerge como estratégia do SUS com base nos princípios de Universalidade, Equidade e Integralidade, sendo assinalada por ações preventivas, diagnósticas e curativas, possibilitando a redução significativa da morbimortalidade materno-infantil quando realizado com qualidade (Brasil, 2011). Entretanto, sua implementação é realizada de forma gradual entre as unidades federativas e municípios, desse modo, contribuinte como fator de risco às condições de saúde, sobretudo, em cidades pertencentes à região Nordeste, uma vez que a implementação de políticas de saúde isoladas não irão, necessariamente, refletir sobre as iniquidades e desigualdades sociais tampouco, causar impacto aos indicadores de saúde, logo, favorecendo a manutenção do perfil encontrado no estudo (Gonçalves et al., 2021; Barbosa et al., 2021).

Esse levantamento pode ser observado em estudos realizados em diferentes cidades do estado de Rio Grande do Norte onde, em consenso com os dados desse estudo, Natal/RN expõe frequência gestante diagnosticada com sífilis durante o parto, com mais de $80 \%$ apenas em 2015; tendência ao aumento de inadequações no esquema de tratamento e mais de $90 \%$ dos parceiros não recebeu tratamento adequado para sífilis (Ferreira et al., 2018). Apesar de não avaliado e tendo como base o não tratamento das gestantes, ficam implícito as lacunas diante ao tratamento oportuno do parceiro, sendo esse um dos maiores problemas existentes ao prognóstico da sífilis (Ferreira et al., 2021). Com isso, além das falhas assistenciais dos serviços ao Pré-Natal citadas anteriormente, autores inferem certa resistência dos profissionais da saúde na administração da Penicilina nas Unidades Básica de Saúde e, como justificativas, remetem ao receio devido à possibilidade de ocorrência das reações anafiláticas, portanto, não administraram na ausência de médicos, além das limitações de recursos e a estrutura das unidades de 
saúde para atender os eventos com maior gravidade (Brasil, 2018; Figueiredo et al., 2020).

Todavia, segundo Domingues et al (2021), os profissionais da saúde devem seguir o protocolo de tratamento para sífilis e respeitar as diretrizes terapêuticas diante a administração da benzilpenicilina benzatina, sendo essa com possibilidade raríssima de reação anafilática em cerca de $0,002 \%$ dos casos, portanto, segura e efetiva. A partir dos dados, corrobora o nível de desinformação entre profissionais da saúde que estão diretamente vinculados a assistência gestacional e a relevância desse cenário diante a elevada frequência da transmissão vertical que, segundo Fiocruz (2016), quando o tratamento é realizado de forma precoce, adequada e seguindo o esquema, é capaz de evitar até $100 \%$ a incidência da sífilis congênita. Portanto, remete a essencialidade das políticas públicas voltadas ao fortalecimento da assistência durante o período gestacional, sobretudo, ao PréNatal, como meta na ampliação do acesso, tratamento, monitoramento e a capacitação dos profissionais da atenção básica com vista na lógica da atenção integral e o manejo adequado da doença. Ademais, sabendo que o Pré-Natal é uma prática fundamentada na APS, a manutenção dos indicadores de ESF e AB são essenciais para solucionar barreiras dispostas aos serviços públicos e assegurar a tríade de vigilância-assistência-prevenção à sífilis e, consequentemente, qualificar a atenção na perspectiva materno-infantil e minimizar a ocorrência de eventos indesejáveis na gestação.

\section{Conclusão}

A partir dos dados, foi observada oscilação na taxa de detecção para sífilis na gestação e congênita durante todo período analisado. Referente ao perfil da gestante diagnosticada com sífilis, houve maior frequência da faixa etária de 20 a 29 anos, com baixa escolaridade, expondo predomínio do nível fundamental incompleto; idade gestacional presente no terceiro trimestre, contudo, expondo transição no perfil da classificação clínica a partir de 2014, saindo da maior frequência de sífilis primária para sífilis latente. No mais, quanto à situação assistencial, foi observado maior frequência na realização do Pré-natal, entretanto, com aumento no diagnóstico durante o parto e pós-parto; além da elevação do diagnóstico inadequado e decréscimo dos indicadores de APS durante o período. Com isso, reconhecendo o desfecho desfavorável da sífilis em gestantes na transmissão vertical e o diagnóstico e tratamento precoce como base para sua diminuição, corrobora a necessidade de melhorias no modelo assistencial da APS disposto na cidade de Parnamirim/RN visando à expansão e ampliação dos serviços e ações descentralizadas e focalizadas na qualidade da atenção em saúde, além de atuações em nível de educação em saúde às gestantes e a capacitação dos profissionais para operar de forma integral e ativa nas UBS.

\section{Referências}

Barbosa, M. M., Chaves, E. C. R., Leite, D, S., Quaresma, A. H. C., Albuquerque, G. P., Costa, I. L. O. F., Reis, A. P. O., Junior, S, A, O., Mendonça, M. H. R\& Lima, S. B. A. (2021). Rede cegonha: avanços e desafios da gestão no ambiente hospitalar. Revista Eletrônica Acervo Saúde, 13(3).

Brasil. (2011). Portaria no 1.459, de 24 de junho de 2011. Institui, no âmbito do Sistema Único de Saúde - SUS - a Rede Cegonha.

Brasil. (2018). Ministério da Saúde. Projeto de Resposta Rápida à Sífilis fortalece debate no RN.

Brasil. (2018). Ministério da Saúde. Implementação do Projeto Resposta Rápida à Sífilis $48^{\text {a }}$ CAMS- RN.

Brasil. (2019). Ministério da Saúde. Pré-Natal.

Brasil. (2019). Ministério da Saúde. Secretaria de Vigilância em Saúde. Departamento de Doenças de Condições crônicas e Infecções Sexualmente Transmissíveis. Protocolo Clínico e Diretrizes Terapêuticas para Prevenção da Transmissão Vertical de HIV, Sífilis e Hepatites Virais.

Brasil. (2020). Ministério da Saúde Secretaria de Vigilância em Saúde Departamento de Doenças de Condições Crônicas e Infecções Sexualmente Transmissíveis. Brasília.

Camargos, M. V \& Oliver, F. C. (2019). Uma experiência de uso do georreferenciamento e do mapeamento no processo de territorialização na Atenção Primária à Saúde. Saúde Debate. Rio de Janeiro, 43(123);1259-1269.

Conceição, H. N., Camara, J. T \& Pereira, B. M (2019). Análise epidemiológica e espacial dos casos de sífilis gestacional e congênita. Saúde Debate, 43(123);1145-1158

Conselho de Secretarias Municipais de Saúde do Rio Grande do Norte - Cosems-RN. (2018). Parnamirim avança no diagnóstico e tratamento da Sífilis. 
Costa, I. L. O. F., Trindade, C. B. S., Chaves, E. C. R., Ferreira, I. P., Lima, S. B. A., Costa, F. B., Mendonça, M. H. R. de, \& Neto, R. L. S. (2020). A vigilância em saúde e o planejamento nas equipes de atenção primária em saúde: revisão narrativa. Revista Eletrônica Acervo Saúde, (53), e3622.

Costa, I. L. O. F., Trindade, C. B. S., Ferreira, I. P., Nunes, S. F., Santos, R. C. S., Lima, V. L. A., Lima, S. B. A \& Chaves, E. C. R. (2021). Vigilância em Saúde \& planejamento e avaliação em Unidades de Saúde da Família: Estudo qualitativo. Research, Society and Development, 10(6).

Domingues, R. M. S. M \& Leal, M. C. (2016). Incidência de sífilis congênita: dados de o estudo nascer no Brasil. Cad. Saúde Pública, Rio de Janeiro, 32(6).

Domingues, C. S. B., Duarte, G., Passos, M. R. L., Sztajnbok, D. C. N \& Menezes, M. L. B. (2021). Protocolo Brasileiro para Infecções Sexualmente Transmissíveis 2020: sífilis congênita e criança exposta à sífilis. Epidemiol. Serv. Saude, Brasília, 30(1).

Donalísio, M. R., Freire, J. B \&Mendes, E. T. (2017). Investigação da sífilis congênita na microrregião de Sumaré, Estado de São Paulo, Brasil-desvelando a fragilidade do cuidado à mulher gestante e ao recém-nascido. Epidemiologia e Serviços de Saúde,16(3):165-73.

Ferreira, A. G., Gomes, L. A., R., Medeiros, C. T., Leonidas, R. A. M \& Medeiros, J. P. (2018). Perfil dos Casos de Sífilis Congênita no Município de Natal / RN no Período de 2007 a 2015. Revista Saúde em Foco, 5(1); 3-27.

Ferreira. F. K. S., Rolim, A. C. A \& Bonfada, D. (2021). Perfil dos casos de sífilis congênita no Rio Grande do Norte: estudo de série temporal. Revista Ciência Plural; 7(2):33-46.

Fundação Oswaldo Cruz - Fiocruz (2016). Tratamento precoce da sífilis.

Figueiredo, D. C. M., Figueiredo, A. M., Souza, T. K. B., Tavares, G \& Vianna, R. P. T. (2020). Relação entre oferta de diagnóstico e tratamento da sífilis na atenção básica sobre a incidência de sífilis gestacional e congênita. Cad. Saúde Pública,36(3).

Freitas, F. L. S., Bensaken, A. S., Passos, M. R. L., Coelho, I. C. B \& Miranda, A. E. (2021). Protocolo Brasileiro para Infecções Sexualmente Transmissíveis 2020: sífilis adquirida. Epidemiol. Serv. Saude, Brasília.

Gonçalves, D. G. N., Mezzomo, E. S., Carvalho, J. R., Souza, L. C. K. \& Mendona, M. H. R. (2021). Avaliação da distribuição espacial da sífilis gestacional e congênita na região norte e nordeste. International Journal of Development Research, 11, (05): 46916-46922.

Instituto Brasileiro de Geografia e Estatística - IBGE (2018). Relatório PNAD: Desigualdade no Brasil e RN.

Junior, S. A. O., Santos, M. B., Silva, E. P., Henrique, G., L., Carvalho, K. R. S., Pereira, K. R. S \& Carrasco, E. (2019) Perfil epidemiológico da sífilis materna e congênita no pré-natal do município de Parnamirim/RN. João Pessoa. Anais do $8^{\circ}$ Congresso Brasileiro de Ciências Sociais e Humanas em Saúde.

Lima, T. M., Machado, I. L. L., Siqueira, J. P \& Almeida, M. T. G (2019). Perfil epidemiológico de pacientes com sífilis congênita e gestacional em um município do Estado de São Paulo, Brasil. Rev. Bras. Saúde Mater. Infant., Recife, 19 (4): 873-880.

Lopes, A. C. (2020). Cortes no orçamento colocam o SUS à beira do colapso. EcoDebate.

Macêdo, V. C., Lira, P. I. c., Frias, P. G., Romaguera, L. M. D., Caires, S. F. F \& Ximenes, R. A. A. (2017). Fatores de risco para sífilis em mulheres: estudo caso-controle. Rev Saude Publica, 51:78.

Macêdo, V. C., Romaguera, L. M. D., Ramalho, M. O. A., Vanderelei, L. C. M., Frias, P. G \& Lira, P. I. C. (2020). Sífilis na gestação: barreiras na assistência pré-natal para o controle da transmissão vertical. Cad. Saúde Colet., 28(4).

Miranda, A. E., Freitas, F. L. S., Passos, M. R. L., Lopes, M. A. A \& Pereira, G. F. M (2021). Políticas públicas em infecções sexualmente transmissíveis no Brasil. Epidemiol. Serv. Saúde, Brasília, 30(1).

Motta, I. A., Delfino, I. R. S., Santos, L. V., Morita, M. O., Gnçalves, R., Gomes, D., Martins, T. P. S., Carellos, E. V. M \& Romaelli, R. C. (2018). Sífilis congênita: por que sua prevalência continua tão alta? Rev Med Minas Gerais, 28.

Neto, L. G., Gomes, L. M \& Sobrinho, H. M. R. (2019). Epidemiologia da sífilis gestacional e congênita no estado de Goiás no período de 2013 a 2018. Revista Brasileira Militar de Ciências, 5(13).

Nunes, J. P. G \& Barbosa, V. V. (2020). Uma série histórica da incidência de Sífilis na gestante no Pará entre os períodos de 2013 a 2018 . Pará Res Med J, 4:e32.

Oliveira, E. H., Holanda, E. C., Silva, L. C., Brito, M. C. S \& Sousa, P. C. M. (2020). Avaliação epidemiológica da sífilis congênita na região Nordeste do Brasil. Research, Society and Development, 10(3).

Organização Mundial da Saúde - OMS. (2017). Comitê Regional para África Estratégia Global para o Setor de Saúde relativa a Infecções Sexualmente Transmiss'veis 2016-2021:quadro de execução para região africana. Relatório do Secretariado.

Pilger, B., Bortoli, M. C. F. C \& Battisti, E. E. S. (2019). Perfil epidemiológico da sífilis congênita em u município do sudoeste do Paraná. Rer. Saúde Pública, $1(2) ; 20-17$

Sanson, M. C. G., Feitoza, H. A. C., Saraceni, V., Koifman, R. J \& Bessa, A. R. S. (2018). Prevalência e perfil epidemiológico da Hepatite B em gestantes: um estudo populacional em uma cidade da Amazônia Ocidental brasileira, no período de 2007 a 2015. Rev. Bras. Saúde Mater. Infant., Recife, 18 (4): $723-733$

Tiago, Z. S., Picoli, R. P., Graeff, S. V., Cunha, R. V \& Arantes, R. (2017). Subnotificação de sífilis em gestantes, congênita e adquirida entre povos indígenas em Mato Grosso do Sul, 2011-2014. Epidemiol. Serv. Saude, Brasília, 26(3):503-512. 